Research Article

\title{
Antimicrobial Resistance in Typhoidal Salmonella in a Tertiary Care Teaching Centre in North India
}

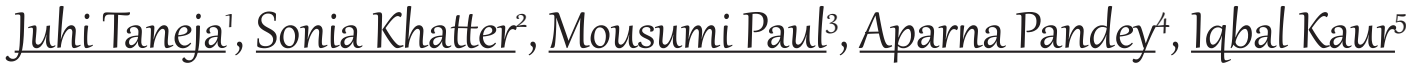 \\ ${ }^{1,3}$ Assistant Professor, ${ }^{2}$ Professor, ${ }^{4}$ Senior Resident, ${ }^{5}$ Professor \& Head, Department of Microbiology, ESIC Medical College \& \\ Hospital NH-3, NIT Faridabad, Haryana, India. \\ DOI: https://doi.org/10.24321/0019.5138.202101
}

\section{I $\quad \mathbf{N} \quad \mathbf{F} \quad \mathbf{O}$}

\section{Corresponding Author:}

Juhi Taneja, Department of Microbiology ESIC Medical College \& Hospital NH-3, NIT Faridabad, Haryana, India.

E-mail Id:

drjuhitaneja@gmail.com

Orcid Id:

https://orcid.org/0000-0001-9622-9034

How to cite this article:

Taneja J, Khatter S, Paul M, Pandey A, Kaur I. Antimicrobial Resistance in Typhoidal Salmonella in a Tertiary Care Teaching Centre in North India. J Commun Dis 2021; 53(1): 1-4.

Date of Submission: 2021-01-11

Date of Acceptance: 2021-01-29

\section{$\begin{array}{llllllll}\mathbf{A} & \mathbf{B} & \mathbf{S} & \mathbf{T} & \mathbf{R} & \mathbf{A} & \mathbf{C} & \mathbf{T}\end{array}$}

Introduction: Enteric fever continues to carry a high burden of morbidity and mortality in India. There have been reports of emergence of ceftriaxone resistant typhoidal Salmonella from Asia. Monitoring of antimicrobial resistance trends in typhoidal Salmonella is crucial to support in clinical decision making.

Aim: To study the current susceptibility pattern of typhoidal salmonella isolates in our setup.

Methods: This retrospective study was conducted on 144 non-repeat blood-culture isolates of S. Typhi, and S. Paratyphi A obtained from 3926 blood cultures received at 510-bedded tertiary-care hospital of NorthIndia from 2017-2019. Identification and antibiograms were obtained by Vitek-2 compact and Kirby-Bauer's disc diffusion with sensitivity to azithromycin, and chloramphenicol.

Result: S. Typhi and S. Paratyphi A in a ratio of 5.2:1 were seen between months of June and July predominantly distributed between 1-10 years age group. S. Typhi resistance to co-trimoxazole, chloramphenicol, ceftriaxone and azithromycin was $21.4 \%, 25.6 \%, 12.3 \%$ and $28 \%$ respectively. S. Paratyphi A resistance to co-trimoxazole, chloramphenicol, ceftriaxone and azithromycin was $4.3 \%, 17.3 \%, 34.7 \%$ and $21.7 \%$ respectively.

Conclusion: Enteric-fever is a major public-health problem in India. Emergence of ceftriaxone-resistant Salmonella mandates appropriate investigation of all febrile illnesses with blood culture whenever possible. Provision of safe drinking water, good sanitation, hygiene and vaccination strategies are needed to sustain herd-immunity.

Keywords: Salmonella Typhi Paratyphi A Ceftriaxone Resistant

\section{Introduction}

Typhoid fever and paratyphoid fever is faeco-orally transmitted disease characterized by high fever and other systemic signs of bacteremia caused by Sal. enterica subspecies enterica Se. Typhi and Se. Paratyphi respectively. Enteric fever if left untreated, the course can be severe and lethal. WHO has recommended risk-based use of typhoid polysaccharide vaccine against S. Typhi. The estimated number of typhoid fever cases in low and middle income countries in 2010 after adjusting for water-related risk was 11.9 million cases with 129,000 deaths. ${ }^{1}$ Currently, third-generation cephalosporins such as ceftriaxone are recommended as first-line therapy. ${ }^{2}$ Enteric fever continues to carry a high burden of morbidity and mortality in India. 
The emergence and rapid spread of ceftriaxone-resistant $S$. Typhi and S. Paratyphi $A$ is alarming. To date, only sporadic reports of ceftriaxone-resistant $S$. Typhi have been published, mainly from Asia (including Japan) but also from West and southern Africa. ${ }^{3}$ As the antimicrobial resistance in typhoid fever has been increasing, this study was designed to study the current susceptibility pattern of typhoidal Salmonella strains in our setup.

\section{Materials and Methods}

We conducted a retrospective analysis of Salmonella isolates from blood cultures received in Microbiology Laboratory at ESIC College \& Hospital Faridabad from Jan 2017-Dec 2019. The study was reviewed and approved by institutional ethics committee. All the blood culture samples of patients (indoor and outdoor) with febrile illness were processed in BacT/ Alert blood culture system (Biomerieux, France). The study isolates were identified and tested for Antimicrobial Resistance (AMR) profiles by VITEK 2 (Biomerieux, France) employing the Clinical and Laboratory Standards Institute (CLSI) guidelines relevant to the year. ${ }^{4}$ In addition, antimicrobial susceptibility was done for azithromycin (15 $\mu \mathrm{g})$ and chloramphenicol (30 $\mu \mathrm{g}$ ) (OXOID) using Kirby-Bauer's disc diffusion method as these antimicrobials are not covered in susceptibility panel for gram negative bacilli in VITEK 2. The laboratory participates in external quality assurance scheme. Serotyping of the strains was done with Denka Seiken (Tokyo, Japan) antisera. E. coli ATCC 25922 was used for quality control.

\section{Result}

A total of 3926 blood cultures were received from January 2017 to December 2019. Out of these, (10\%) 395 blood cultures were positive. A total of (36.4\%) 144 isolates were identified as Sal. enterica during the study period. Out of these, 121 (84\%) S. Typhi, and 23 (15.9\%) were S. Paratyphi A. Serotyping showed complete concordance with identification by VITEK 2. Most of the salmonellae were isolated in the months of June and July, with the majority being in the 1-10 years followed by $21-30$ years age groups with a male preponderance. Table 1, shows that the percentage antimicrobial resistance for ciprofloxacin in S. Typhi was $87.6 \%$. Similarly in S. Paratyphi A, ciprofloxacin resistance was 82.6\% (Table 2). Ceftriaxone, cotrimoxazole, azithromycin, chloramphenicol resistance was detected in S. Typhi as $12.3 \%, 21.4 \%, 28 \%$ \& $25.6 \%$ respectively (Table 1 ). Whereas resistance to ceftriaxone, cotrimoxazole, azithromycin, chloremphenicol in S. Paratyphi A was 34.7\%, 4.3\%, 21.7\%, $17.3 \%$ respectively (Table 2 ). All the isolates were sensitive to meropenem. No multidrug-resistant Salmonella strains were isolated.

Table I.Antimicrobial resistance patterns obtained for S. Typhi isolates recovered from blood cultures of patients between 2017 and $2019(n=121)$

\begin{tabular}{|c|c|c|c|}
\hline Antimicrobial agents & Sensitive No. (\%) & Intermediate No. (\%) & Resistant No. (\%) \\
\hline Ampicillin & $93(76.8)$ & - & $28(23.1)$ \\
\hline Ciprofloxacin & $15(12.4)$ & 23 & $83(68.5)$ \\
\hline Nalidixic acid & 0 & - & $121(100)$ \\
\hline Sulfamethoxazole/Trimethoprim & $95(78.5)$ & - & $26(21.4)$ \\
\hline Ceftriaxone & $95(78.5)$ & $11(9)$ & $15(12.3)$ \\
\hline Azithromycin & $87(71.9)$ & - & $34(28)$ \\
\hline Chloramphenicol & $90(74.3)$ & - & $31(25.6)$ \\
\hline Meropenem & $121(100)$ & 0 & 0 \\
\hline
\end{tabular}

Table 2.Antimicrobial resistance patterns obtained for S. Paratyphi A isolates recovered from blood cultures of patients between 2017 and $2019(n=23)$

\begin{tabular}{|c|c|c|c|}
\hline Antimicrobial agents & Sensitive No. (\%) & Intermediate No. (\%) & Resistant No. (\%) \\
\hline Ampicillin & - & $5(21.7)$ & $18(78.2)$ \\
\hline Ciprofloxacin & $4(17.3)$ & - & $19(82.6)$ \\
\hline Nalidixic acid & 0 & - & 23 (100) \\
\hline Sulfamethoxazole/ Trimethoprim & $22(95.6)$ & - & $1(4.3)$ \\
\hline Ceftriaxone & $10(43.4)$ & $4(17.3)$ & $5(34.7)$ \\
\hline Azithromycin & $18(78.2)$ & - & $4(21.7)$ \\
\hline Chloramphenicol & $19(82.6)$ & - & $4(17.3)$ \\
\hline Meropenem & $23(100)$ & 0 & 0 \\
\hline
\end{tabular}




\section{Discussion}

Enteric fever continues to be a major public health burden in India. There have been emerging reports of the recent change of AMR pattern of typhoidal Salmonella from India. A high percentage of blood cultures (10\%) grew typhoidal Salmonella. In this study, S. Typhi was the most common serotype (84\%) similar to the results of other Indian studies. ${ }^{5,7,8}$

The preferred test for assessing fluoroquinolone susceptibility or resistance in Salmonella spp. is a ciprofloxacin MIC test. ${ }^{4}$ All the 144 isolates tested were $86.8 \%$ resistant to fluoroqinolones. High-level fluoroquinolone resistance has been reported in Salmonella isolates in various studies ranging from $84.5 \%$ to $95.5 \% .^{6,8}$ In view of development of high level of Fluoroquinolone resistance, third generation cephalosporins and azithromycin are currently the preferred drugs for treatment of enteric fever leading to their widespread use.

As per recent National treatment guidelines for antimicrobial use in infectious diseases, Azithromycin or Cefixime is recommended as a monotherapy for uncomplicated enteric fever in outpatient settings while Ceftriaxone is the drug of choice where parenteral therapy required for inpatients. ${ }^{2}$ Concurrent with their use, reports of resistance to resistance to third generation cephalosporins have emerged leading to treatment failures. Bhattacharya et al, in 2011 study from Orissa reported resistance of 3.0 to 6.25 per cent against third generation cephalosporins among the salmonella isolates. ${ }^{7}$ Makkar A et al. in a study from Delhi reported $3.86 \%, 17.2 \%$ resistance to third generation cephalosporins in S. Typhi \& S. Paratyphi A respectively. ${ }^{8}$ On similar lines, we found $12.3 \% \mathrm{~S}$. Typhi and a higher percentage of $S$. Paratyphi A (34.7\%) strains ceftriaxone resistant in this study. However, other studies from north India did not report any resistance to ceftriaxone. ${ }^{9,10}$

Member of the macrolide class, Azithromycin is a very effective and convenient oral option for treatment of typhoid fever in adults and children. In several Indian studies, susceptibility of Salmonella serotypes to azithromycin has been documented varying from $91-92 \% .{ }^{11}$ Dutta et al in a study from Kolkata reported sensitivity of $72 \%$ in Sal. Paratyphi $A$ isolates. ${ }^{12}$ We report similar pattern in our study, as $71.9 \%$ S. Typhi and $78.2 \%$ S. Paratyphi were sensitive to azithromycin.

$76.8 \%$ of the S. Typhi isolates were sensitive to ampicillin, $78.5 \%$ of them to co-trimoxazole and $74.3 \%$ to chloramphenicol. Similarly, $21.7 \%$ of the S. Paratyphi A isolates were intermediate to ampicillin, $95.6 \%$ of them sensitive to co-trimoxazole and $82.6 \%$ sensitive to chloramphenicol. None of the isolate was MDR. Non-susceptibility of Typhoidal Salmonella to first-line antimicrobials (chloramphenicol, co- trimoxazole and ampicillin) has decreased over time as also reported by other studies. ${ }^{5,6,13,14}$ Hence routine surveillance of resistance determinants is essential to understand the comeback of first generation of antibiotics for the treatment of typhoid fever.

Ceftriaxone is commonly used as monotherapeutic agent for treatment of typhoid fever. Resistance to ceftriaxone and azithromycin has complicated treatment of typhoid infection. The treatment options available for resistance isolates are fourth generation cephalosporins, carbapenems, and tigecycline or combination antibiotic therapy. The study shows a worrying increase of ceftriaxone resistant isolates. There is a need to ramp up typhoid vaccination in pediatric population and adhere to safe food and water practices. Management of acute febrile illness should be guided by appropriate investigation including blood culture whenever possible.

\section{Conflict of Interest: None \\ References}

1. Mogasale V, Maskery B, Ochiai RL et al. Burden of typhoid fever in low-income and middle-income countries: a systematic, literature-based update with risk-factor adjustment. Lancet Glob Health 2014; 2(10): e570-80.

2. National Treatment Guidelines for Antimicrobial Use in Infectious Diseases 2016. National Centre for Disease Control, Directorate General of Health Services Ministry of Health \& Family Welfare Government of India.

3. Crump JA, Sjölund-Karlsson M, Gordon MA et al. Epidemiology, clinical presentation, laboratory diagnosis, antimicrobial resistance, and antimicrobial management of invasive salmonella infections. Clin Microbiol Rev 2015; 28(4): 901-937.

4. Clinical and Laboratory Standards Institute. M100. Performance Standard for Antimicrobial Susceptibility Testing: Thirtieth Informational Supplement. Clinical and Laboratory Standard Institute, Wayne, PA. 2020.

5. Harichandran D, Dinesh KR. Antimicrobial susceptibility profile, treatment outcome and serotype distribution of clinical isolates of Salmonella enterica subspecies enterica: a 2-year study from Kerala, South India. Infect Drug Resist 2017; 10: 97-101.

6. Singhal L, Gupta PK, Kale P et al. Trends in antimicrobial susceptibility of Salmonella Typhi from North India (2001-2012). Indian J Med Microbiol 2014; 32(2): 14952.

7. Bhattacharya SS, Das U, Choudhury BK. Occurrence \& antibiogram of Salmonella Typhi \& S. Paratyphi A isolated from Rourkela, Orissa. Indian J Med Res 2011; 133(4): 431-433.

8. Makkar A, Gupta S, Khan ID et al. Epidemiological profile and antimicrobial resistance pattern of enteric 
fever in a tertiary care hospital of North India - a Seven Year Ambispective Study. Acta Medica (Hradec Kralove) 2018; 61(4): 125-130.

9. Wattal C, Goel N. Pediatric blood cultures and antibiotic resistance: an overview. Indian J Pediatr 2020; 87(2): 125-131.

10. Sharma P, Dahiya S, Manral N et al. Changing trends of culture-positive typhoid fever and antimicrobial susceptibility in a tertiary care North Indian Hospital over the last decade. Indian J Med Microbiol 2018; 36(1): 70-76.

11. Patel SR, Bharti S, Pratap CB et al. Drug resistance pattern in the recent isolates of salmonella typhi with special reference to cephalosporins and azithromycin in the gangetic plain. J Clin Diagn Res 2017; 11(6): DM01-3.

12. Dutta S, Das S, Mitra U et al. Antimicrobial resistance, virulence profiles and molecular subtypes of Salmonella enterica serovars Typhi and Paratyphi A blood isolates from Kolkata, India during 2009-2013. PLoS One 2014; 9(8): e101347.

13. Patil N, Mule P. Sensitivity pattern of salmonella typhi and paratyphi an isolates to chloramphenicol and other anti-typhoid drugs: an in vitro study. Infect Drug Resist 2019; 12: 3217-25.

14. Britto CD, John J, Verghese VP et al. A systematic review of antimicrobial resistance of typhoidal Salmonella in India. Indian J Med Res 2019; 149(2): 151-163. 